\title{
On the optimal asset allocation strategy for a defined contri- bution pension system with refund clause of premium with predetermined interest under Heston's volatility model
}

\author{
Edikan E. Akpanibah ${ }^{\mathrm{a}}$, Bright O. Osu ${ }^{\mathrm{b}, *}$, Silas A. Ihedioha ${ }^{\mathrm{c}}$ \\ ${ }^{a}$ Department of Mathematics and Statistics, Federal University Otuoke, P.M.B 126, Yenagoa, Bayelsa State, Nigeria. \\ ${ }^{b}$ Department of Mathematics, Michael Okpara University of Agriculture, Umudike, Abia State, Nigeria. \\ ${ }^{c}$ Department of Mathematics, Plateau State University Bokkos, P.M.B 2012 Jos, Plateau state, Nigeria.
}

\begin{abstract}
In this paper, we study optimal asset allocation strategy for a defined contribution (DC) pension fund with return of premium clause under Heston's volatility model in mean-variance utility frame work. In this model, members' next of kin are allowed to withdraw their family members' accumulated premium with predetermined interest. Also, investments in one risk free asset and one risky asset are considered to help increase the accumulated funds of the remaining members in order to meet their retirement needs. Using the actuarial symbol, we formulize the problem as a continuous time mean-variance stochastic optimal control problem. We establish an optimization problem from the extended Hamilton Jacobi Bellman equations using the game theoretic approach and solve the optimization problem to obtain the optimal allocation strategy for the two assets, the optimal fund size and also the efficient frontier of the pension members. We analyze numerically the effect of some parameters on the optimal allocation strategy and deduce that as the initial wealth, predetermined interest rate and risk averse level increases, the optimal allocation policy for the risky asset (equity) decreases. Furthermore, we give a theoretical comparison of our result with an existing result and observed that the optimal allocation policy whose return is with predetermined interest is higher compared to that without predetermined interest.
\end{abstract}

Keywords: DC pension fund, extended HJB equation, optimal allocation policy, refund of contribution clause, interest rate. 2010 MSC: 91G10, 91B30.

(C)2020 All rights reserved.

\section{Introduction}

In general, a man's life cycle is made up of two crucial phase; first, the person's working years and his retirement years. Based on this, individuals need to create a definite consumption plan for their needs from when income is earned (working life) to when there might be no other funds available, except for a possible survival level of support from the members' accumulated contribution during working life or members employers contributions (retirement life). The funds given to a retiree monthly from the accumulated contributions after their working years is referred to as pension. The important of pension

\footnotetext{
${ }^{*}$ Corresponding author

Email addresses: edikanakpanibah@gmail.com (Edikan E. Akpanibah), osu.bright@mouau.edu.ng (Bright O. Osu)
}

doi: $10.22436 /$ jnsa.013.01.05

Received: 2018-12-17 Revised: 2019-05-19 Accepted: 2019-06-03 
scheme cannot be over emphasized in the life of retirees. There are two types of pension plan in which members can take part in; this include the defined benefit (DB) pension plan and the defined contribution pension plan (DC). The defined benefit pension plan is a type in which members benefits are determined in advance following some basic requirements which include age, years in service, members' salary histories etc., these benefits depend basically on the contributions made by the employers and because of the mode of contributions, most private organizations found it difficult to develop a pension plan for their members as a result, this plan was limited to members in government organizations. Although most members are happy with this plan since contributions are made only by the employers, it has over the years generated controversies and delay in implementation after retirement and these has led to the introduction of the alternative plan known as the defined contribution (DC) pension plan which is mostly members dependent. It also requires that members contribute a certain proportion of their income into the members' retirement serving account (RSA). The DC pension plan is much more attractive and reliable than the DB pension plan since members are fully involved in the contribution and investment process and depend mostly on the returns of the investment during the accumulation period and this expected return is influenced by some factors such as investment efficiency, inflation, mortality risk, etc.. Although the DC pension plan seems attractive, it requires investment knowledge in different assets available in the financial market. These assets include the cash, bond, and stock etc.. Since investment in stock involve risk, there is need to study the best possible way to invest for optimal returns. This leads to the study of optimal allocation policy by financial institutions and this explains the proportion of the members' wealth to be invested in various assets available in the financial market for optimal returns with less risk. In [6], a model of optimal allocation for DC pension plan with a minimum guarantee in the continuous-time setting was proposed and investigated. [5], studied optimal investment strategy to DC members with asset, salary and interest rate risk and proposed a novel form of terminal utility function by incorporating habit formulation. [4] worked on optimal investment strategy for a DC pension with stochastic interest rate. In [7], asset allocation problem under a stochastic interest rate was studied, and [2], investigated a case where the interest rate was of Vasicek model, [18] studied optimal investment strategies for DC pension with a stochastic salary under affine interest rate model which includes the Cox-Ingersoll-Ross (CIR) model and Vasicek model. Lately, the study of constant elasticity of Variance (CEV) model in DC pension fund investment strategies have taken centre stage in modelling the stock price. [16], studied the constant elasticity of variance (CEV) model and the Legendre transform-dual solution for annuity contracts. [8], obtained explicit solutions of the optimal investment strategy for investor with CRRA and CARA utility function by extending the work of [16]. [14], studied optimal investment strategies in DC pension fund with multiple contributions using Legendre transformation method to obtain the explicit solution for CRRA and CARA. [1], studied stochastic strategies of optimal investment for DC pension fund with multiple contributors where they considered the rate of contribution to be stochastic. [13], studied optimization problem with return of premium in a DC pension with multiple contributors. The study optimal allocation strategy with refund of contributions clause include [10], who studied optimal investment strategy for a defined contribution pension scheme with the return of premiums clauses in a mean-variance utility function. [11] investigated equilibrium investment strategy for DC pension plan with default risk and return of premiums clauses under constant elasticity of variance model. [15], investigated the optimal time-consistent investment strategy for a DC pension with the return of premiums clauses and annuity contracts. To the best of our knowledge, there is no literature that has any work done on optimal allocation strategy with refund of contributions clause that considers the refund contributions with predetermined interest under Heston volatility model. This forms the basis of our discussion in this paper.

\section{Mathematical formulation}

Consider a financial market which is complete and frictionless and is continuously open over a fixed period of time interval $0 \leqslant t \leqslant T$, where $T$ is the retirement age of any given plan member. 
Assume the market is made of a risk-free asset (cash) and a risky asset (equity) and suppose $(\Omega, F, P)$ is a complete probability space such that $\Omega$ is a real space and $\mathrm{P}$ a probability measure satisfying the condition $0 \leqslant t \leqslant T .\left\{W_{s}(t), W_{l}(t): t \geqslant 0\right\}$ are two standard Brownian motions. $\mathcal{F}$ is the filtration and denotes the information generated by the two Brownian motions. Let $C_{t}^{1}(t)$ denote the price of the risk free asset and $C_{t}^{2}(t)$ the price of the risky asset which satisfies the Heston's stochastic volatility model. Their price models are described as follows:

$$
\begin{aligned}
& \frac{d C_{t}^{1}(t)}{C_{t}^{1}(t)}=r_{1} d t, \quad C_{t}^{1}(0)=1, \\
& \frac{d C_{t}^{2}(t)}{C_{t}^{2}(t)}=\left(r_{1}+k_{1} L(t)\right) d t+\sqrt{L(t)} d W_{1} C_{t}^{2}(0)=c_{0}^{2}, \\
& \frac{d L(t)}{L(t)}=m\left(\frac{\theta}{L}-1\right) d t+\sigma_{1} \frac{\sqrt{L}}{L} d W_{2}, \quad L(0)=l_{0} .
\end{aligned}
$$

Here $r_{1}$ is the predetermine interest rate of the risk free asset and $m, \theta, k_{1}, \sigma_{1}$ are positive constants and the two Brownian motions $W_{1}(t), W_{2}(t)$ are such that $E\left[W_{1}(t), W_{2}(t)\right]=\rho$, where $\rho$ represents the correlation coefficient of the two Brownian motions and satisfies the condition $-1 \leqslant \rho \leqslant 1$.

Assume the premium received at a given time be represented as $b$, let $\pi_{0}$ represent the initial age of accumulation phase, $T$, the period of the accumulation phase such that $\pi_{0}+T$ is the end age. The actuarial symbol $\mathfrak{M}_{\left(\frac{1}{i}\right), \pi_{0}+t}$ is the mortality rate from time $t$ to $t+\frac{1}{i}$, bt is the premium accumulated at time $t, t b \mathfrak{M}_{\left(\frac{1}{i}\right), \pi_{0}+t}$ is the premium returned to the death members. Secondly, we assume that apart from the accumulated fund of the death member, a certain fixed interest is paid as well from the investment in the risk-free asset since the risk free interest rate is predetermined. Let $\varphi$ represent the proportion of the wealth invested in risky asset and $\varphi_{1}=1-\varphi$ is the proportion invested in riskless asset.

Considering the time interval $\left[t, t+\frac{1}{i}\right]$, the differential form associated with the fund size is given as:

$$
\begin{aligned}
& Z\left(t+\frac{1}{i}\right)=\left(\begin{array}{c}
Z(t)\left(\varphi_{1} \frac{C_{t+\frac{1}{i}}^{1}(t)}{C_{t}^{1}}+\varphi \frac{C_{t+\frac{1}{i}}^{2}(t)}{C_{t}^{2}}\right)+ \\
b\left(\frac{1}{i}\right)-t b \frac{1}{i} \mathfrak{M}_{\vartheta_{0}+t}-\varphi_{1} Z(t) \frac{C_{t+\frac{1}{i}}^{1}(t)}{C_{t}^{1}} \frac{1}{i} \mathfrak{M}_{\vartheta_{0}+t}
\end{array}\right) \frac{1}{1-\frac{1}{i} \mathfrak{M}_{\vartheta_{0}+t}}, \\
& \mathrm{Z}\left(\mathrm{t}+\frac{1}{i}\right)=\left(\begin{array}{c}
\mathrm{Z}(\mathrm{t})\left(\begin{array}{c}
1+(1-\varphi)\left(\frac{\mathrm{C}_{\mathrm{t}+\frac{1}{i}}^{1}-\mathrm{C}_{\mathrm{t}}^{1}}{\mathrm{C}_{\mathrm{t}}^{1}}\right. \\
+\varphi\left(\frac{\mathrm{C}_{\mathrm{t}+\frac{1}{i}}^{2}-\mathrm{C}_{\mathrm{t}}^{2}}{\mathrm{C}_{\mathrm{t}}^{2}}\right) \\
+\mathrm{b}\left(\frac{1}{i}\right)-\mathrm{tb} \frac{1}{i} \mathfrak{M}_{\vartheta_{0}+\mathrm{t}} \\
-(1-\varphi) Z(\mathrm{t})\left(1+\frac{\mathrm{C}_{\mathrm{t}+\frac{1}{i}}^{1}-\mathrm{C}_{\mathrm{t}}^{1}}{\mathrm{C}_{\mathrm{t}}^{1}}\right)
\end{array}\right) \frac{1}{i} \mathfrak{M}_{\vartheta_{0}+\mathrm{t}}
\end{array}\right)\left(1+\frac{\frac{1}{i} \mathfrak{M}_{\vartheta_{0}+\mathrm{t}}}{1-\frac{1}{i} \mathfrak{M}_{\vartheta_{0}+\mathrm{t}}}\right)
\end{aligned}
$$

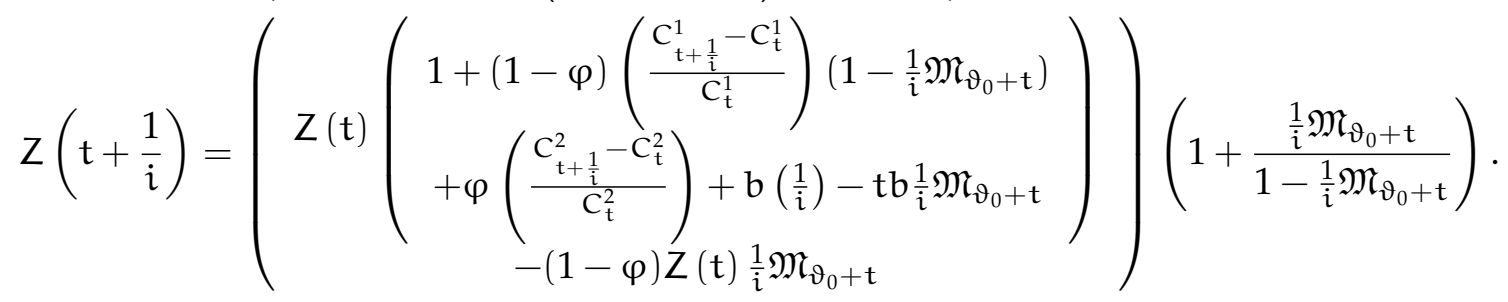

The conditional death probability ${ }_{t} q_{x}=1-{ }_{t} p_{x}=1-e^{-\int_{0}^{t} \pi\left(\vartheta_{0}+t+s\right) d s}$, where $\pi(t)$ is the force function 
of the mortality at time $t$, and for $i \rightarrow \infty$,

$$
\begin{aligned}
\frac{1}{\mathfrak{i}} \mathfrak{M}_{\vartheta_{0}+t} & =1-\exp \left\{-\int_{0}^{\frac{1}{i}} \pi\left(\vartheta_{0}+t+s\right) d s\right\} \approx \pi\left(\vartheta_{0}+t\right) \frac{1}{i}=O\left(\frac{1}{i}\right), \\
\frac{\frac{1}{i} \mathfrak{M}_{\vartheta_{0}+t}}{1-\frac{1}{i} \mathfrak{M}_{\vartheta_{0}+t}} & =\frac{1-\exp \left\{-\int_{0}^{\frac{1}{i}} \pi\left(\vartheta_{0}+t+s\right) d s\right\}}{\exp \left\{-\int_{0}^{\frac{1}{i}} \pi\left(\vartheta_{0}+t+s\right) d s\right\}} \\
& =\exp \left\{\int_{0}^{\frac{1}{i}} \pi\left(\vartheta_{0}+t+s\right) d s\right\}-1 \approx \pi\left(\vartheta_{0}+t\right) \frac{1}{i}=O\left(\frac{1}{i}\right), \\
i & \rightarrow \infty, \frac{\frac{1}{i} \mathfrak{M}_{\vartheta_{0}+t}}{1-\frac{1}{i} \mathfrak{M}_{\vartheta_{0}+t}}=\pi\left(\vartheta_{0}+t\right) d t, \frac{1}{i} \mathfrak{M}_{\vartheta_{0}+t}=\pi\left(\vartheta_{0}+t\right) d t b, \\
\left(\frac{1}{i}\right) & \rightarrow b d t, \frac{C_{t+\frac{1}{i}}^{1}-C_{t}^{1}}{C_{t}^{1}} \rightarrow \frac{d C_{t}^{1}(t)}{C_{t}^{1}(t)}, \frac{C_{t+\frac{1}{i}}^{2}-C_{t}^{2}}{C_{t}^{2}} \rightarrow \frac{d C_{t}^{2}(t)}{C_{t}^{2}(t} .
\end{aligned}
$$

Substituting (2.4) into (2.3), we have

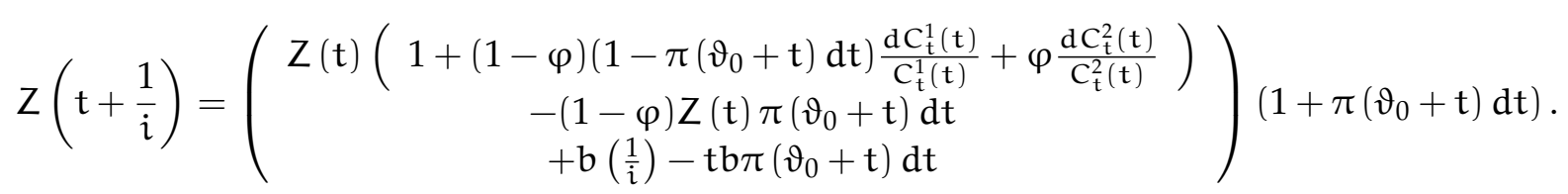

Substituting (2.1) and (2.2) into (2.5), we have

$d Z(t)=\left(\left\{Z(t)\left(\varphi\left(k_{1} L(t)+\frac{1}{\vartheta-\vartheta_{0}-t}\right)+r_{1}\right)+b\left(\frac{\vartheta-\vartheta_{0}-2 t}{\vartheta-\vartheta_{0}-t}\right)\right\} d t+Z(t)\left(\varphi \sqrt{L(t)} d W_{1}\right), \quad Z(0)=z_{0}\right.$, where $\vartheta$ is the maximal age of the life table and $\pi(t)$ is the force function given by

$$
\pi(t)=\frac{1}{\vartheta-t}, \quad 0 \leqslant t<\vartheta, \quad \pi\left(\vartheta_{0}+t\right)=\frac{1}{\vartheta-\vartheta_{0}-t} .
$$

\section{Methodology}

If we consider the pension wealth and the volatility of the accumulations, the remaining members of the pension scheme will want to increase their total wealth and minimize the risk as much as possible. Hence there is need to formulate the optimal portfolio problem under the mean-variance criterion as follows:

$$
\sup _{\varphi}\left\{E_{t, z, l} Z^{\varphi}(T)-\operatorname{Var}_{t, z, l} Z^{\varphi}(T)\right\} .
$$

Our main aim is to obtain the optimal investment strategies for both the risk-free and risky asset using the mean-variance utility function. Applying game theoretic method in $[3,10]$ the mean-variance control problem in (3.1) is equivalent to the following Markovian time inconsistent stochastic optimal control problem with value function $\mathrm{C}(\mathrm{t}, z, \mathrm{l})$

$$
\left\{\begin{array}{l}
D(t, z, l, \varphi)=E_{t, z, l}\left[Z^{\varphi}(T)\right]-\frac{\gamma}{2} \operatorname{Var}_{t, z, l}\left[Z^{\varphi}(T)\right], \\
D(t, z, l, \varphi)=E_{t, z, l}\left[Z^{\varphi}(T)\right]-\frac{\gamma}{2}\left(E_{t, z, l}\left[Z^{\varphi}(T)^{2}\right]-\left(E_{t, z, l}\left[Z^{\varphi}(T)\right]\right)^{2}\right), \\
C(t, z, l)=\sup _{\varphi} D(t, z, l, \varphi) .
\end{array}\right.
$$

Following [15], the optimal portfolio policy $\varphi$ satisfies: $C(t, z, l)=\sup _{\varphi} D\left(t, z, l, \varphi^{*}\right) . \quad \gamma$ is a constant representing risk aversion coefficient of the members. Let $p^{\varphi}(t, z, l)=E_{t, z, l}\left[Z^{\varphi}(T)\right], q^{\varphi}(t, z, l)=$ $E_{t, z, l}\left[Z^{\varphi}(T)^{2}\right]$, then $C(t, z, l)=\sup _{\varphi} u\left(t, z, l, p^{\varphi}(t, z, l), q^{\varphi}(t, z, l)\right)$, where

$$
u(t, z, l, p, q)=p-\frac{\gamma}{2}\left(q-p^{2}\right) .
$$


Theorem 3.1 (Verification theorem). If there exist three real functions $\mathrm{U}, \mathrm{V}, \mathrm{W}:[0, \mathrm{~T}] \times \mathrm{R} \rightarrow \mathrm{R}$ satisfying the following Hamilton Jacobi Bellman equations:

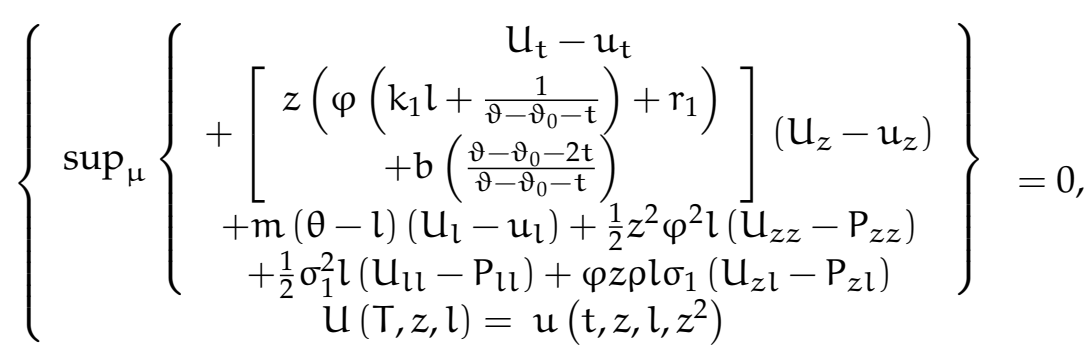

where

$$
\begin{aligned}
& \left\{\begin{array}{c}
\mathrm{P}_{z z}=u_{z z}+2 u_{z p} p_{z}+2 u_{z q} q_{z}+u_{p p} p_{z}^{2}+2 u_{p q} p_{z} q_{z}+u_{q q} q_{z}^{2}=\gamma V_{z}^{2}, \\
P_{l l}=u_{l l}+2 u_{l p} p_{l}+2 u_{l q} q_{l}+u_{p p} p_{l}^{2}+2 u_{p q} p_{l} q_{l}+u_{q q} q_{l}^{2}=\gamma V_{l}^{2}, \\
P_{z l}=u_{z l}+u_{z p} p_{l}+u_{z q} q_{l}+u_{p l} p_{z}+u_{q l} q_{z}+u_{p p} p_{z} p_{l} \\
\quad+u_{p q} p_{z} q_{l}+u_{p q} p_{l} q_{z}+u_{q q} q_{l} q_{z}=\gamma V_{z} V_{l},
\end{array}\right. \\
& \left\{+\left[\begin{array}{c}
z\left(\varphi\left(k_{1} l+\frac{1}{\vartheta-\vartheta_{0}-t}\right)+r_{1}\right) \\
+b\left(\frac{\vartheta-\vartheta_{0}-2 t}{\vartheta-\vartheta_{0}-t}\right) \\
+m(\theta-l) V_{l}+\frac{1}{2} z^{2} \varphi^{2} l V_{z z} \\
+\frac{1}{2} \sigma_{1}^{2} l V_{l l}+\varphi z \rho l \sigma_{1} V_{z l}
\end{array}\right\} \mathrm{V}_{z}\right\}=0, \\
& \mathrm{~V}(\mathrm{~T}, z, \mathrm{l})=z \text {, }
\end{aligned}
$$

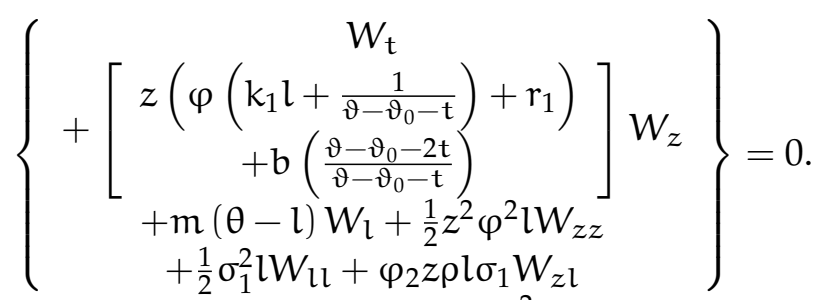

$$
\begin{aligned}
& \mathrm{W}(\mathrm{T}, z, \mathrm{l})=z^{2} \text {, }
\end{aligned}
$$

Then $\mathrm{C}(\mathrm{t}, \mathrm{z}, \mathrm{l})=\mathrm{U}(\mathrm{t}, z, \mathrm{l}), \mathrm{p}^{\varphi^{*}}=\mathrm{V}(\mathrm{t}, z, \mathrm{l}), \mathrm{q}^{\varphi^{*}}=\mathrm{W}(\mathrm{t}, \mathrm{z}, \mathrm{l})$ for the optimal investment strategy $\varphi^{*}$.

The details of the proof can be found in $[9,12,17]$. Next, we try to find the optimal allocation strategies for the two assets and also the efficient frontier by solving (3.2), (3.3), and (3.4).

Proposition 3.2. The optimal allocation policy for equity is given as

$$
\begin{aligned}
\varphi_{1}{ }^{*}=1-\varphi^{*} & =1-\frac{e^{r_{1}(t-T)}}{\gamma z}\left\{k_{1}\left[1-\frac{\rho \sigma_{1} k_{1}}{m+\rho \sigma_{1} k_{1}}\left(1-e^{\left(m+\rho \sigma_{1} k_{1}\right)(t-T)}\right)\right]+\frac{1}{l}\left(\frac{1}{\vartheta-\vartheta_{0}-t}\right)\right\}, \\
\varphi^{*} & =\frac{e^{r_{1}(t-T)}}{\gamma z}\left\{k_{1}\left[1-\frac{\rho \sigma_{1} k_{1}}{m+\rho \sigma_{1} k_{1}}\left(1-e^{\left(m+\rho \sigma_{1} k_{1}\right)(t-T)}\right)\right]+\frac{1}{l}\left(\frac{1}{\vartheta-\vartheta_{0}-t}\right)\right\} .
\end{aligned}
$$

Proof. Recall that $u(t, z, l, p, q)=p-\frac{\gamma}{2}\left(q-p^{2}\right)$,

$$
\begin{aligned}
& u_{t}=u_{z}=u_{l}=u_{z z}=u_{l l}=u_{z l}=u_{p l}=u_{q l}=u_{z p}=u_{z q}=u_{p q}=u_{q q}=0, \\
& u_{p}=1+\gamma p, \quad u_{p p}=\gamma, \quad u_{q}=-\frac{\gamma}{2} .
\end{aligned}
$$

Substituting (3.5) into (3.2) and differentiating it with respect to $\varphi$ and solving for $\varphi$, we have:

$$
\varphi^{*}=-\left[\frac{\left(\mathrm{k}_{1} \mathrm{l}+\frac{1}{\vartheta-\vartheta_{0}-\mathrm{t}}\right) \mathrm{u}_{z}+\left(\mathrm{U}_{z l}-\gamma \mathrm{V}_{z} \mathrm{~V}_{\mathrm{l}}\right) \rho \sigma_{1}}{z \mathrm{l}\left(\mathrm{U}_{z z}-\gamma \mathrm{V}_{z}^{2}\right)}\right]
$$


Substituting (3.6) and (3.5) into (3.2) and (3.3) we have

$$
\begin{aligned}
& u_{t}+\left[r_{1} z+b\left(\frac{\vartheta-\vartheta_{0}-2 t}{\vartheta-\vartheta_{0}-t}\right)\right] u_{z}+m(\theta-l) u_{l}+\frac{1}{2}\left(u_{l l}-\gamma v_{l}^{2}\right) \sigma_{1}^{2} l \\
& -\frac{1}{2} \frac{\mathrm{U}_{z}^{2}}{\left(\mathrm{U}_{z z}-\gamma \mathrm{V}_{z}^{2}\right)}\left(\frac{\left(\mathrm{k}_{1} \mathrm{l}+\frac{1}{\vartheta-\vartheta_{0}-\mathrm{t}}\right)^{2}}{l}\right)-\frac{1}{2} \frac{\left(\mathrm{U}_{z l}-\gamma \mathrm{V}_{z} \mathrm{~V}_{\mathrm{l}}\right)^{2}}{\left(\mathrm{U}_{z z}-\gamma \mathrm{V}_{z}^{2}\right)} \rho^{2} \sigma_{1}^{2}=0, \\
& V_{t}+V_{z}\left[r_{1} z+b\left(\frac{\vartheta-\vartheta_{0}-2 t}{\vartheta-\vartheta_{0}-t}\right)\right]+m(\theta-l) V_{l}+\frac{1}{2} \sigma_{1}^{2} l V_{l l}-\frac{u_{z} V_{z}}{\left(u_{z z}-\gamma V_{z}^{2}\right)}\left(\frac{\left(k_{1} l+\frac{1}{\vartheta-\vartheta_{0}-t}\right)^{2}}{l}\right) \\
& -\frac{\mathrm{V}_{z}\left(\mathrm{U}_{z l}-\gamma \mathrm{V}_{z} \mathrm{~V}_{\mathrm{l}}\right)}{\left(\mathrm{U}_{z z}-\gamma \mathrm{V}_{z}^{2}\right)}\left(\mathrm{k}_{1} \mathrm{l}+\frac{1}{\vartheta-\vartheta_{0}-\mathrm{t}}\right) \rho \sigma_{1} \\
& +\frac{1}{2}\left(l\left(\frac{\left(k_{1} l+\frac{1}{\vartheta-\vartheta_{0}-t}\right) u_{z}+\left(\mathrm{U}_{z l}-\gamma \mathrm{V}_{z} \mathrm{~V}_{\mathrm{l}}\right) \rho \sigma_{1}}{l\left(\mathrm{U}_{z z}-\gamma \mathrm{V}_{z}^{2}\right)}\right)^{2}\right) \mathrm{v}_{z z} \\
& +\rho \sigma_{1}\left(\frac{\left(k_{1} l+\frac{1}{\vartheta-\vartheta_{0}-t}\right) u_{z}+\left(\mathrm{U}_{z l}-\gamma \mathrm{V}_{z} \mathrm{~V}_{\mathrm{l}}\right) \rho \sigma_{1}}{\left(\mathrm{U}_{z z}-\gamma \mathrm{V}_{z}^{2}\right)}\right) \mathrm{V}_{\mathrm{l} z}=0
\end{aligned}
$$

We will next assume a solution for $U(t, z, l)$ and $V(t, z, l)$ as follows:

$$
\left\{\begin{array}{l}
\mathrm{U}(\mathrm{t}, z, l)=X_{1}(\mathrm{t}) z+\frac{X_{2}(t) l}{\gamma}+\frac{X_{3}(t)}{\gamma}, X_{1}(T)=1, X_{2}(T)=0, X_{3}(T)=0 \\
V(t, z, l)=Y_{1}(t) z+\frac{Y_{2}(t) l}{\gamma}+\frac{Y_{3}(t)}{\gamma}, Y_{1}(T)=1, Y_{2}(T)=0, \quad Y_{3}(T)=0 \\
U_{t}=z \frac{d X_{1}(t)}{d t}+\frac{l}{\gamma} \frac{d X_{2}(t)}{d t}+\frac{1}{\gamma} \frac{d X_{3}(t)}{d t}, U_{z}=X_{1}(t), U_{z z}=0, U_{l}=\frac{X_{2}(t)}{\gamma}, U_{l l}=0 \\
V_{t}=z \frac{d Y_{1}(t)}{d t}+\frac{l}{\gamma} \frac{d Y_{2}(t)}{d t}+\frac{1}{\gamma} \frac{d Y_{3}(t)}{d t}, V_{z}=Y_{1}(t), V_{z z}=0, V_{l}=\frac{Y_{2}(t) l}{\gamma}, V_{l l}=0
\end{array}\right.
$$

Substituting (3.9) into (3.7) and (3.8), we have

$$
\begin{aligned}
& {\left[\frac{d X_{1}(t)}{d t}+r_{1} X_{1}(t)\right] z+\left[\frac{d X_{2}(t)}{d t}-m X_{2}+\frac{\left(\rho^{2}-1\right) \sigma_{1}^{2} Y_{2}^{2}}{2}+\frac{k_{1}^{2} X_{1}^{2}}{2 Y_{1}^{2}}-\frac{\rho \sigma_{1} k_{1} X_{1} Y_{2}}{Y_{1}}\right] \frac{l}{\gamma}} \\
& +\left[\frac{d X_{3}(t)}{d t}+m \theta X_{2}+X_{1} b \gamma\left(\frac{\vartheta-\vartheta_{0}-2 t}{\vartheta-\vartheta_{0}-t}\right)-\left(\frac{1}{\vartheta-\vartheta_{0}-t}\right) \rho \sigma_{1} k_{1} Y_{1}\right. \\
& \left.+\left(\frac{1}{\vartheta-\vartheta_{0}-t}\right) k_{1} \frac{X_{1}^{2}}{Y_{1}^{2}}+\frac{1}{2} \frac{\left(\frac{1}{\vartheta-\vartheta_{0}-t}\right)^{2} X_{1}^{2}}{l Y_{1}^{2}}\right] \frac{1}{\gamma}=0 \\
& \left\{\begin{array}{l}
\frac{d X_{1}(t)}{d t}+r_{1} X_{1}(t)=0, \\
\frac{d X_{2}(t)}{d t}-m X_{2}+\frac{\left(\rho^{2}-1\right) \sigma_{1}^{2} Y_{2}^{2}}{2}+\frac{k_{1}^{2}}{2}-\rho \sigma_{1} k_{1} Y_{2}=0, \\
\frac{d X_{3}(t)}{d t}+m \theta X_{2}+X_{1} b \gamma\left(\frac{\vartheta-\vartheta_{0}-2 t}{\vartheta-\vartheta_{0}-t}\right)-\left(\frac{1}{\vartheta-\vartheta_{0}-t}\right) \rho \sigma_{1} k_{1} Y_{1}+\left(\frac{1}{\vartheta-\vartheta_{0}-t}\right) k_{1}+\frac{1}{2 l}\left(\frac{1}{\vartheta-\vartheta_{0}-t}\right)^{2}=0,
\end{array}\right. \\
& {\left[\frac{d Y_{1}(t)}{d t}+r_{1} Y_{1}(t)\right] z+\left[\frac{d Y_{2}(t)}{d t}-m Y_{2}+\frac{k_{1}^{2} X_{1}}{Y_{1}}-\frac{\rho \sigma_{1} k_{1} X_{1} Y_{2}}{Y_{1}}\right] \frac{l}{\gamma}}
\end{aligned}
$$




$$
\begin{gathered}
+\left[\frac{d Y_{3}(t)}{d t}+m \theta Y_{2}+Y_{1} b \gamma\left(\frac{\vartheta-\vartheta_{0}-2 t}{\vartheta-\vartheta_{0}-t}\right)-\left(\frac{1}{\vartheta-\vartheta_{0}-t}\right) \rho \sigma_{1} k_{1} Y_{2}\right. \\
\left.+2 k_{1}\left(\frac{1}{\vartheta-\vartheta_{0}-t}\right) \frac{X_{1} Y_{1}}{Y_{1}^{2}}+\frac{1}{2}\left(\frac{1}{\vartheta-\vartheta_{0}-t}\right)^{2} \frac{X_{1} Y_{1}}{l Y_{1}^{2}}\right] \frac{1}{\gamma}=0 \\
\left\{\begin{array}{l}
\frac{d Y_{1}(t)}{d t}+r_{1} Y_{1}(t)=0 \\
\frac{d Y_{2}(t)}{d t}-m Y_{2}+k_{1}^{2}-\rho \sigma_{1} k_{1} Y_{2}=0, \\
\frac{d Y_{3}(t)}{d t}+m \theta Y_{2}+Y_{1} b \gamma\left(\frac{\vartheta-\vartheta_{0}-2 t}{\vartheta-\vartheta_{0}-t}\right)-\left(\frac{1}{\vartheta-\vartheta_{0}-t}\right) \rho \sigma_{1} k_{1} Y_{2}+2 k_{1}\left(\frac{1}{\vartheta-\vartheta_{0}-t}\right)+\frac{1}{2 l}\left(\frac{1}{\vartheta-\vartheta_{0}-t}\right)^{2}=0
\end{array}\right.
\end{gathered}
$$

Solving (3.10) and (3.11), we have

$$
\begin{aligned}
& X_{1}(t)=e^{r_{1}(T-t)} \\
& X_{2}(t)=\frac{\rho \sigma_{1} k_{1}^{3}}{m+\rho \sigma_{1} k_{1}}\left\{\begin{array}{c}
\frac{1}{m}\left(e^{m(t-T)}-1\right) \\
+\frac{1}{\rho \sigma_{1} k_{1}}\left(e^{m(t-T)}-e^{\left(m+\rho \sigma_{1} k_{1}\right)(t-T)}\right)
\end{array}\right\}+\frac{k_{1}^{2}}{2 m}\left(1-e^{m(t-T)}\right) \\
& +\frac{\sigma_{1}^{2} k_{1}^{3}\left(\rho^{2}-1\right)}{2\left(m+\rho \sigma_{1} k_{1}\right)^{2}}\left\{\begin{array}{c}
\frac{1}{m}\left(e^{m(t-T)}-1\right) \\
+\frac{2 e^{m(t-T)}}{\rho \sigma_{1} k_{1}}\left(1-e^{\rho \sigma_{1} k_{1}(t-T)}\right)+ \\
\frac{e^{m(t-T)}}{m+2 \rho \sigma_{1} k_{1}}\left(e^{\left(m+\rho \sigma_{1} k_{1}\right)(t-T)}-1\right)
\end{array}\right\} \\
& X_{3}(t)=-m \theta \int_{t}^{T} X_{2}(\tau) d \tau+b \gamma \int_{t}^{T} \frac{\tau e^{r_{1}(T-\tau)}}{\vartheta-\vartheta_{0}-\tau} d \tau+\frac{b \gamma}{r_{1}}\left(e^{r_{1}(t-T)}-1\right)+\left(\begin{array}{c}
k_{1} \\
+\frac{1}{2 l\left(\vartheta-\vartheta_{0}-t\right)\left(\vartheta-\vartheta_{0}-T\right)}
\end{array}\right)(T-t), \\
& Y_{1}(t)=e^{r_{1}(T-t)}, \\
& Y_{2}(t)=\frac{k_{1}^{2}}{m+\rho \sigma_{1} k_{1}}\left(1-e^{\left(m+\rho \sigma_{1} k_{1}\right)(t-T)}\right), \\
& Y_{3}(t)=-m \theta \int_{t}^{T} Y_{2}(\tau) d \tau-\rho \sigma_{1} k_{1} \int_{t}^{T}\left(\frac{1}{\vartheta-\vartheta_{0}-t}\right) Y_{2}(\tau) d \tau+b \gamma \int_{t}^{T} \frac{\tau e^{r_{1}(T-\tau)}}{\vartheta-\vartheta_{0}-\tau} d \tau \\
& +\left(\frac{k_{1}^{2}}{m+\rho \sigma_{1} k_{1}}\right) \int_{t}^{T} \frac{e^{\left(m+\rho \sigma_{1} k_{1}\right)(T-\tau)}}{\vartheta-\vartheta_{0}-\tau} d \tau+\frac{b \gamma}{r_{1}}\left(e^{r_{1}(t-T)}-1\right) \\
& +\frac{1}{l}\left(\frac{1}{2\left(\vartheta-\vartheta_{0}-t\right)\left(\vartheta-\vartheta_{0}-T\right)}\right)(T-t)+\left(2 k_{1}-\frac{k_{1}^{2}}{m+\rho \sigma_{1} k_{1}}\right) \ln \left(\frac{\vartheta-\vartheta_{0}-T}{\vartheta-\vartheta_{0}-t}\right) \text {, } \\
& u(t, z, l)=z e^{r_{1}(T-t)}+\frac{l}{\gamma}\left[+\frac{\sigma_{1}^{2} k_{1}^{3}\left(\rho^{2}-1\right)}{2\left(m+\rho \sigma_{1} k_{1}\right)^{2}}\left\{\begin{array}{c}
+\frac{k_{1}^{2}}{2 m}\left(1-e^{m(t-T)}\right) \\
\frac{1}{m}\left(e^{m(t-T)}-1\right) \\
+\frac{2 e^{m(t-T)}}{\rho \sigma_{1} k_{1}}\left(1-e^{\rho \sigma_{1} k_{1}(t-T)}\right)+ \\
\frac{e^{m(t-T}}{m+2 \rho \sigma_{1} k_{1}}\left(e^{\left(m+\rho \sigma_{1} k_{1}\right)(t-T)}-1\right)
\end{array}\right\}\right)
\end{aligned}
$$

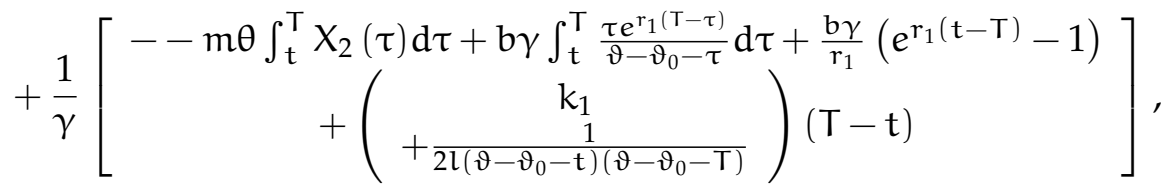

$$
\begin{aligned}
& V(t, z, l)=z e^{r_{1}(T-t)}+\frac{l}{\gamma}\left\{\frac{k_{1}^{2}}{m+\rho \sigma_{1} k_{1}}\left(1-e^{\left(m+\rho \sigma_{1} k_{1}\right)(t-T)}\right)\right\}
\end{aligned}
$$




$$
+\frac{1}{\gamma}\left\{\begin{array}{c}
-m \theta \int_{t}^{T} Y_{2}(\tau) d \tau-\rho \sigma_{1} k_{1} \int_{t}^{T}\left(\frac{1}{\vartheta-\vartheta_{0}-t}\right) Y_{2}(\tau) d \tau \\
+b \gamma \int_{t}^{T} \frac{\tau e^{r_{1}(T-\tau)}}{\vartheta-\vartheta_{0}-\tau} d \tau+\left(\frac{k_{1}^{2}}{m+\rho \sigma_{1} k_{1}}\right) \int_{t}^{T} \frac{e^{\left(m+\rho \sigma_{1} k_{1}\right)(T-\tau)}}{\vartheta-\vartheta_{0}-\tau} d \tau \\
+\frac{b \gamma}{r_{1}}\left(e^{r_{1}(t-T)}-1\right)+\frac{1}{l}\left(\frac{1}{2\left(\vartheta-\vartheta_{0}-t\right)\left(\vartheta-\vartheta_{0}-T\right)}\right)(T-t) \\
+\left(2 k_{1}-\frac{k_{1}^{2}}{m+\rho \sigma_{1} k_{1}}\right) \ln \left(\frac{\vartheta-\vartheta_{0}-T}{\vartheta-\vartheta_{0}-t}\right) \\
u_{z}=X_{1}(t), V_{z}=Y_{1}(t), u_{z z}=0 \text {, and } V_{l}=Y_{2}(t) .
\end{array}\right.
$$

Substituting (3.12) into (3.6), we have

$$
\begin{aligned}
\varphi_{1}^{*}=1-\varphi^{*} & =1-\frac{e^{r_{1}(t-T)}}{\gamma z}\left\{k_{1}\left[1-\frac{\rho \sigma_{1} k_{1}}{m+\rho \sigma_{1} k_{1}}\left(1-e^{\left(m+\rho \sigma_{1} k_{1}\right)(t-T)}\right)\right]+\frac{1}{l}\left(\frac{1}{\vartheta-\vartheta_{0}-t}\right)\right\} \\
\varphi^{*} & =\frac{e^{r_{1}(t-T)}}{\gamma z}\left\{k_{1}\left[1-\frac{\rho \sigma_{1} k_{1}}{m+\rho \sigma_{1} k_{1}}\left(1-e^{\left(m+\rho \sigma_{1} k_{1}\right)(t-T)}\right)\right]+\frac{1}{l}\left(\frac{1}{\vartheta-\vartheta_{0}-t}\right)\right\} .
\end{aligned}
$$

Proposition 3.3. The efficient frontier of the pension members is given by

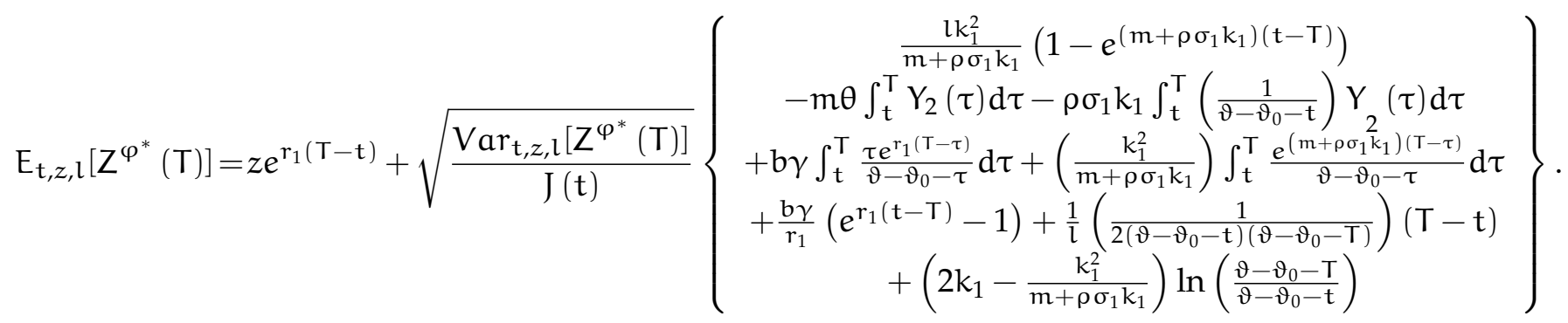

Proof.

$$
\begin{aligned}
& \operatorname{Var}_{t, z, l}\left[Z^{\varphi^{*}}(T)\right]=E_{t, z, l}\left[Z^{\varphi^{*}}(T)^{2}\right]-\left(E_{t, z, l}\left[Z^{\varphi^{*}}(T)\right]\right)^{2}=\frac{2}{\gamma}(V(t, z, l)-U(t, z, l)) \\
& =\frac{1}{\gamma^{2}}\left[2 l\left\{\begin{array}{c}
\frac{\rho \sigma_{1} k_{1}^{3}}{m+\rho \sigma_{1} k_{1}}\left\{\begin{array}{c}
\frac{k_{1}^{2}}{m+\rho \sigma_{1} k_{1}}\left(1-e^{\left(m+\rho \sigma_{1} k_{1}\right)(t-T)}\right) \\
+\frac{1}{m}\left(e^{m(t-T)}-1\right) \\
\left.+\sigma_{1}^{m(t-T)}-e^{\left(m+\rho \sigma_{1} k_{1}\right)(t-T)}\right)
\end{array}\right\} \\
+\frac{k_{1}^{2}}{2 m}\left(1-e^{m(t-T)}\right) \\
+\frac{\sigma_{1}^{2} k_{1}^{3}\left(\rho^{2}-1\right)}{2\left(m+\rho \sigma_{1} k_{1}\right)^{2}}\left\{\begin{array}{c}
\frac{1}{m}\left(e^{m(t-T)}-1\right) \\
+\frac{2 e^{m(t-T)}}{\rho \sigma_{1} k_{1}}\left(1-e^{\rho \sigma_{1} k_{1}(t-T)}\right)+ \\
\frac{e^{m(t-T)}}{m+2 \rho \sigma_{1} k_{1}}\left(e^{\left(m+\rho \sigma_{1} k_{1}\right)(t-T)}-1\right)
\end{array}\right\}
\end{array}\right\}\right] \\
& +\left\{\begin{array}{c}
-m \theta \int_{t}^{T}\left(Y_{2}(\tau)+X_{2}(\tau)\right) d \tau \\
-\rho \sigma_{1} k_{1} \int_{t}^{T}\left(\frac{1}{\vartheta-\vartheta_{0}-t}\right) Y_{2}(\tau) d \tau \\
+\left(\frac{k_{1}^{2}}{m+\rho \sigma_{1} k_{1}}\right) \int_{t}^{T} \frac{\left.e^{\left(m+\rho \sigma_{1} k_{1}\right.}\right)(T-\tau)}{\vartheta-\vartheta_{0}-\tau} d \tau \\
k_{1}(T-t) \\
+\left(2 k_{1}-\frac{k_{1}^{2}}{m+\rho \sigma_{1} k_{1}}\right) \ln \left(\frac{\vartheta-\vartheta_{0}-T}{\vartheta-\vartheta_{0}-t}\right)
\end{array}\right\}, \\
& \operatorname{Var}_{\mathrm{t}, \mathrm{x}}\left[\mathrm{Z}^{\varphi^{*}}(\mathrm{~T})\right]=\frac{1}{\gamma^{2}} \mathrm{~J}(\mathrm{t}),
\end{aligned}
$$


where

$$
\begin{aligned}
& J(t)=\left[2 l\left\{\begin{array}{c}
\frac{k_{1}^{2}}{m+\rho \sigma_{1} k_{1}}\left(1-e^{\left(m+\rho \sigma_{1} k_{1}\right)(t-T)}\right) \\
\frac{1}{m}\left(e^{m(t-T)}-1\right) \\
\frac{\rho \sigma_{1} k_{1}^{3}}{m+\rho \sigma_{1} k_{1}}\left\{\begin{array}{c}
(t) \\
+\frac{1}{\rho \sigma_{1} k_{1}}\left(e^{m(t-T)}-e^{\left(m+\rho \sigma_{1} k_{1}\right)(t-T)}\right)
\end{array}\right\}+\frac{k_{1}^{2}}{2 m}\left(1-e^{m(t-T)}\right) \\
+\frac{\sigma_{1}^{2} k_{1}^{3}\left(\rho^{2}-1\right)}{2\left(m+\rho \sigma_{1} k_{1}\right)^{2}}\left\{\begin{array}{c}
\frac{1}{m}\left(e^{m(t-T)}-1\right) \\
+\frac{2 e^{m(t-T)}}{\rho \sigma_{1} k_{1}}\left(1-e^{\rho \sigma_{1} k_{1}(t-T)}\right)+ \\
\frac{e^{m(t-T)}}{m+2 \rho \sigma_{1} k_{1}}\left(e^{\left(m+\rho \sigma_{1} k_{1}\right)(t-T)}-1\right)
\end{array}\right\}
\end{array}\right\}\right. \\
& +\left\{\begin{array}{c}
-m \theta \int_{t}^{T}\left(Y_{2}(\tau)+X_{2}(\tau)\right) d \tau-\rho \sigma_{1} k_{1} \int_{t}^{T}\left(\frac{1}{\vartheta-\vartheta_{0}-t}\right) Y_{2}(\tau) d \tau \\
+\left(\frac{k_{1}^{2}}{?^{\prime}+\rho \sigma_{1} k_{1}}\right) \int_{t}^{T} \frac{e^{\left(m+\rho \sigma_{1} k_{1}\right)(T-\tau)}}{\vartheta-\vartheta_{0}-\tau} d \tau k_{1}(T-t)+\left(2 k_{1}-\frac{k_{1}^{2}}{m+\rho \sigma_{1} k_{1}}\right) \ln \left(\frac{\vartheta-\vartheta_{0}-T}{\vartheta-\vartheta_{0}-t}\right)
\end{array}\right\}, \\
& \frac{1}{\gamma}=\frac{\sqrt{\operatorname{Var}_{t, z,[}\left[Z^{\varphi^{*}}(\mathrm{~T})\right]}}{\sqrt{\mathrm{J}(\mathrm{t})]}} \\
& E_{t, z, l}\left[Z^{\varphi^{*}}(T)\right]=V(t, z, l)=z e^{r_{1}(T-t)}+\frac{1}{\gamma}\left\{\begin{array}{c}
\frac{l k_{1}^{2}}{m+\rho \sigma_{1} k_{1}}\left(1-e^{\left(m+\rho \sigma_{1} k_{1}\right)(t-T)}\right) \\
-m \theta \int_{t}^{T} Y_{2}(\tau) d \tau-\rho \sigma_{1} k_{1} \int_{t}^{T}\left(\frac{1}{\vartheta-\vartheta_{0}-t}\right) Y_{2}(\tau) d \tau \\
+b \gamma \int_{t}^{T} \frac{\tau e^{r_{1}(T-\tau)}}{\vartheta-\vartheta_{0}-\tau} d \tau+\left(\frac{k_{1}^{2}}{m+\rho \sigma_{1} k_{1}}\right) \int_{t}^{T} \frac{e^{\left(m+\rho \sigma_{1} k_{1}\right)(T-\tau)}}{\vartheta-\vartheta_{0}-\tau} d \tau \\
+\frac{b \gamma}{r_{1}}\left(e^{r_{1}(t-T)}-1\right)+\frac{1}{l}\left(\frac{1}{2\left(\vartheta-\vartheta_{0}-t\right)\left(\vartheta-\vartheta_{0}-T\right)}\right)(T-t) \\
+\left(2 k_{1}-\frac{k_{1}^{2}}{m+\rho \sigma_{1} k_{1}}\right) \ln \left(\frac{\vartheta-\vartheta_{0}-T}{\vartheta-\vartheta_{0}-t}\right)
\end{array}\right\} .
\end{aligned}
$$

Substituting (3.13) in (3.14), we have

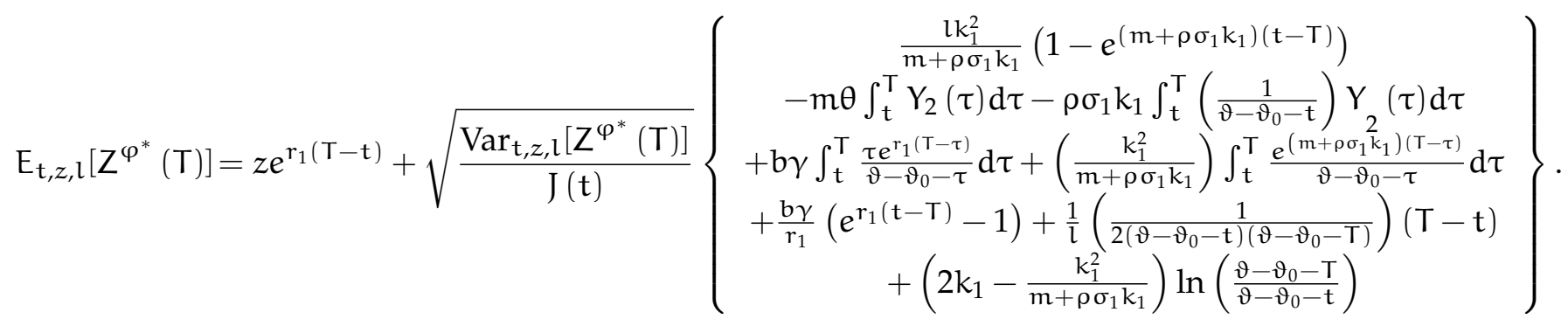

Proposition 3.4. The optimal fund size $\mathrm{Z}^{\varphi^{*}}(\mathrm{t})$ corresponding to the optimal allocation strategy $\varphi^{*}$ is given as

$$
\begin{aligned}
Z(t)= & \frac{\vartheta m k_{1}}{\gamma}\left[\begin{array}{c}
\left(\left(\frac{t}{m}-\frac{1}{m^{2}}\right) e^{m t}+\frac{1}{m^{2}}\right)\left(1-\frac{\rho \sigma_{1} k_{1}}{m+\rho \sigma_{1} k_{1}}\right) e^{r_{1}(t-T)} \\
+e^{m t}\left(\frac{t}{2 m+\rho \sigma_{1} k_{1}}-\frac{1}{\left(2 m+\rho \sigma_{1} k_{1}\right)^{2}}\right) e^{\left(r_{1}+m+\rho \sigma_{1} k_{1}\right)(t-T)} \\
+\left(\frac{1}{\left(2 m+\rho \sigma_{1} k_{1}\right)^{2}} e^{-\left(m+\rho \sigma_{1} k_{1}\right) T} e^{r_{1}(t-T)}\right)
\end{array}\right] \\
& +\frac{k_{1}^{2}}{m \gamma}\left[\begin{array}{c}
e^{r_{1}(t-T)}\left(1-\frac{\rho \sigma_{1} k_{1}}{m\left(m+\rho \sigma_{1} k_{1}\right)}\right) \\
+\frac{1}{2 m+\rho \sigma_{1} k_{1}} e^{\left(r_{1}+m+\rho \sigma_{1} k_{1}\right)(t-T)}
\end{array}\right]\left(e^{m t}-1\right) \\
& +z_{0} e^{r_{1} t}+\frac{b}{m}\left(e^{r_{1} t}-1\right)-b \gamma e^{r_{1} t} \int_{t}^{T} \frac{\tau e^{-r_{1} t}}{\vartheta-\vartheta_{0}-\tau} d \tau .
\end{aligned}
$$




\section{Theoretical analysis}

In this section, we present a proposition comparing our result with the result in [15]. Let $\mu^{*}$ be the optimal asset allocation in [15] and is given as

$$
\mu^{*}=\frac{e^{r_{1}(t-T)}}{\gamma z^{1}}\left\{k_{1}\left[1-\frac{\rho \sigma_{1} k_{1}}{m+\rho \sigma_{1} k_{1}}\left(1-e^{\left(m+\rho \sigma_{1} k_{1}\right)(t-T)}\right)\right]\right\} .
$$

Proposition 4.1. Let $\mathrm{k}_{1}>0, \vartheta>0, \vartheta_{0}>0, \mathrm{r}_{1}>0, \mathrm{~m}>0, \gamma>0, \sigma_{1}>0, \mathrm{~T}>0, \mathrm{t}>0, \rho \in[-1,1]$, and $l(t)>0, l^{1}>0$ for $t \in[0, T]$, such that $z^{1}(t)>z(t)$ and $m+\rho \sigma_{1} k_{1}>0,\left(\vartheta-\vartheta_{0}-t\right)>0$, then $\varphi^{*}>\mu^{*}$.

Proof. Recall that $\varphi^{*}=\frac{e^{r_{1}(t-T)}}{\gamma z}\left\{k_{1}\left[1-\frac{\rho \sigma_{1} k_{1}}{m+\rho \sigma_{1} k_{1}}\left(1-e^{\left(m+\rho \sigma_{1} k_{1}\right)(t-T)}\right)\right]+\frac{1}{l}\left(\frac{1}{\vartheta-\vartheta_{0}-t}\right)\right\}$ and $\mu^{*}=\frac{e^{r_{1}(t-T)}}{\gamma z^{1}}$ $\left\{k_{1}\left[1-\frac{\rho \sigma_{1} k_{1}}{m+\rho \sigma_{1} k_{1}}\left(1-e^{\left(m+\rho \sigma_{1} k_{1}\right)(t-T)}\right)\right]\right\}$. Suppose $\varphi^{*}>\mu^{*}$, we want to show that $\varphi^{*}-\mu^{*}>0$.

$$
\varphi^{*}-\mu^{*}=\frac{1}{\gamma z l}\left(\frac{1}{\vartheta-\vartheta_{0}-t}\right) e^{r_{1}(t-T)}+\frac{\left(z^{1}-z\right)}{z^{1} z} \frac{e^{r_{1}(t-T)}}{\gamma}\left\{k_{1}\left[1-\frac{\rho \sigma_{1} k_{1}}{m+\rho \sigma_{1} k_{1}}\left(1-e^{\left(m+\rho \sigma_{1} k_{1}\right)(t-T)}\right)\right]\right\} .
$$

Since $\left(\vartheta-\vartheta_{0}-t\right)>0,0<e^{r_{1}(t-T)}<1, z l>0$, then

$$
\frac{1}{\gamma z l}\left(\frac{1}{\vartheta-\vartheta_{0}-t}\right) e^{r_{1}(t-T)}>0
$$

Also, $z^{1}-z>0, z^{1} z>0, t-T<0$, then $\frac{\left(z^{1}-z\right)}{z^{1} z} \frac{e^{r_{1}(t-T)}}{\gamma}>0$ and $0<\left(1-e^{\left(m+\rho \sigma_{1} k_{1}\right)(t-T)}\right)<1$.

Case 1. If $\rho=0$, then

$$
\begin{aligned}
& \varphi^{*}-\mu^{*}=\frac{1}{\gamma z l}\left(\frac{1}{\vartheta-\vartheta_{0}-t}\right) e^{r_{1}(t-T)}+\frac{\left(z^{1}-z\right)}{z^{1} z} \frac{e^{r_{1}(t-T)}}{\gamma}\left\{k_{1}\left[1-\frac{\rho \sigma_{1} k_{1}}{m+\rho \sigma_{1} k_{1}}\left(1-e^{\left(m+\rho \sigma_{1} k_{1}\right)(t-T)}\right)\right]\right\} \\
& \varphi^{*}-\mu^{*}=\frac{1}{\gamma z l}\left(\frac{1}{\vartheta-\vartheta_{0}-t}\right) e^{r_{1}(t-T)}+\frac{k_{1}\left(z^{1}-z\right)}{z^{1} z} \frac{e^{r_{1}(t-T)}}{\gamma}>0 .
\end{aligned}
$$

Case 2. If $\rho<0$, then

$\varphi^{*}-\mu^{*}=\frac{1}{\gamma z l}\left(\frac{1}{\vartheta-\vartheta_{0}-t}\right) e^{r_{1}(t-T)}+\frac{\left(z^{1}-z\right)}{z^{1} z} \frac{e^{r_{1}(t-T)}}{\gamma}\left\{k_{1}\left[1-\frac{\rho \sigma_{1} k_{1}}{m+\rho \sigma_{1} k_{1}}\left(1-e^{\left(m+\rho \sigma_{1} k_{1}\right)(t-T)}\right)\right]\right\}>0$.

Since $\frac{\rho \sigma_{1} k_{1}}{m+\rho \sigma_{1} k_{1}}<1$.

Case 3. If $\rho>0$, then

$\varphi^{*}-\mu^{*}=\frac{1}{\gamma z l}\left(\frac{1}{\vartheta-\vartheta_{0}-t}\right) e^{r_{1}(t-T)}+\frac{\left(z^{1}-z\right)}{z^{1} z} \frac{e^{r_{1}(t-T)}}{\gamma}\left\{k_{1}\left[1-\frac{\rho \sigma_{1} k_{1}}{m+\rho \sigma_{1} k_{1}}\left(1-e^{\left(m+\rho \sigma_{1} k_{1}\right)(t-T)}\right)\right]\right\}>0$.

Since $\left[1-\frac{\rho \sigma_{1} k_{1}}{m+\rho \sigma_{1} k_{1}}\left(1-e^{\left(m+\rho \sigma_{1} k_{1}\right)(t-T)}\right)\right]>0$. Therefore

$$
\varphi^{*}-\mu^{*}>0 \text {. }
$$

\section{Numerical simulations}

Here, we give numerical simulations of the optimal investment strategy with respect to time and study the impact of the different parameters of the optimal portfolio strategy with the help of math lab programming language.

The following parameters were used unless otherwise stated $\gamma=0.2, \mathrm{r}_{1}=0.06, \mathrm{~m}=0.5, \rho=0.2, \mathrm{k}_{1}=$ $0.05, \mathrm{l}_{0}=0.1, z_{0}=0.1, \mathrm{~T}=40, \mathrm{t}=0: 5: 20, \vartheta=100, \vartheta_{0}=20$. 


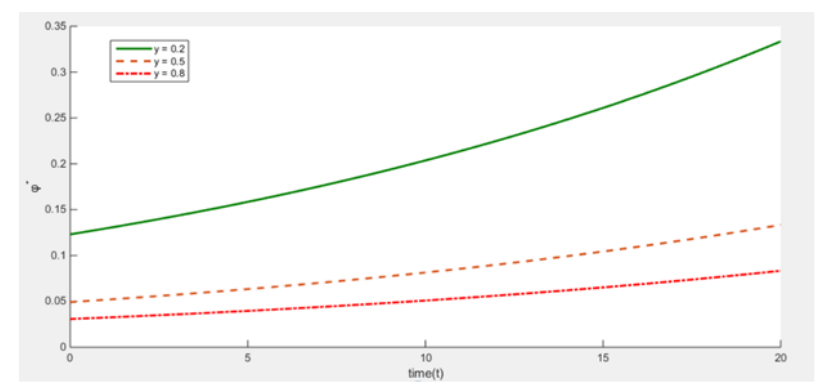

Figure 1: Evolution of optimal allocation strategy with different risk averse level.

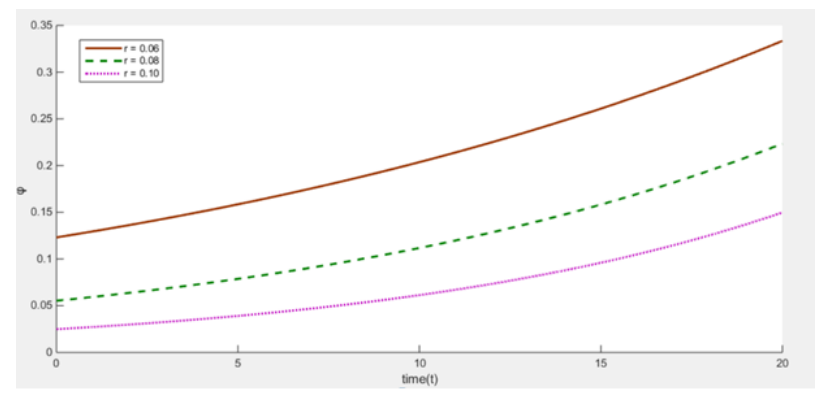

Figure 2: Evolution of optimal allocation strategy with different predetermined interest rates.

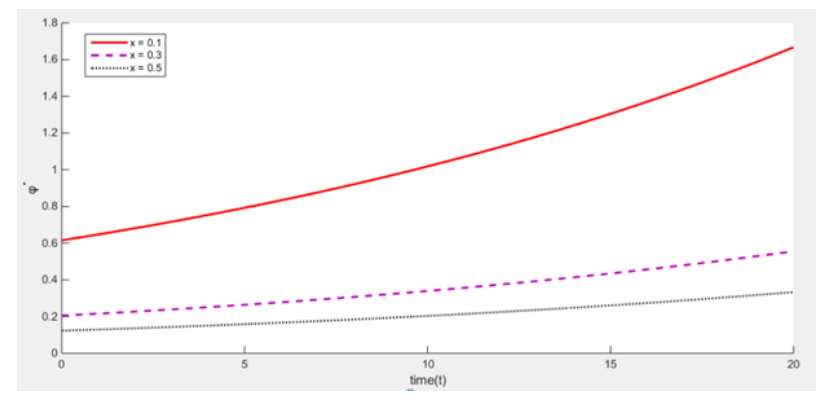

Figure 3: Evolution of optimal allocation strategy with different initial wealth.

\section{Discussion}

From Proposition 4.1, we observed that the optimal allocation strategy whose return of premium is with predetermined interest is greater than that without predetermined interest. The reason being that when the return is with interest, the remaining fund in the pension system is less compared to when the return is without interest and comparing that with Figure 3, we observed that a decrease in wealth leads to an increase in the optimal allocation strategy of the risky assets. This implies that fund manager in case where the return is with interest will take more risk in investing in risky asset with the aim to increase the wealth of the remaining members.

In Figure 1, the optimal allocation strategy increases with a decrease in the risk-averse coefficient. The implication is that members with high risk averse will prefer to invest more in cash and will reduce that of the equity. Figure 2 shows that the optimal allocation strategy increases with a decrease in the interest rate of cash. This implies that if the interest rate of the cash is high, the members will increase the proportion of its wealth to be invested in cash thereby reducing the proportion invested in equity and vice versa. Also, Figure 3, shows that the optimal allocation strategy decreases with increase in the initial wealth. The implication here is that if the initial wealth of the plan member is high, the member will prefer to invest more in cash to minimize risk instead of investing more in equity but if the initial wealth is low, the member prefers taking the risk to grow the wealth by investing in equity. 
In general, we observe that at the beginning of the accumulation phase, the pension manager will invest more in cash because there is no return initially, but once refund is made to the death members, the fund manager will increase its investment in the equity to meet the retirement needs of the remaining members.

\section{Conclusion}

We investigated asset allocation strategy in a defined contribution (DC) pension plan with refund of premium clauses under Heston's Volatility model using mean-variance utility function. We assumed that the refund contributions are with predetermined interest and considered investments in cash and equity to help increase the accumulated funds of the remaining members to meet their retirement needs. We established an optimized problem from the extended Hamilton Jacobi Bellman equations and solved the optimized problem and obtained the optimal allocation strategy for both cash and equity and also the efficient frontier of the members. We compared our result with that in [15]. Next, we analyzed numerically the effect of some parameters on the optimal allocation strategy. Our conclusion is that as the initial wealth, predetermined interest rate, and risk averse level increase, the optimal allocation strategy for the risky asset (equity) decreases.

\section{References}

[1] E. E. Akpanibah, S. K. Samaila, Stochastic strategies for optimal investment in a defined contribution (DC) pension fund, Int. J. Appl. Sci. Math. Theory, 3 (2017), 48-55. 1

[2] P. Battocchio, F. Menoncin, Optimal pension management in a stochastic framework, Insurance Math. Econom., 34 (2004), 79-95. 1

[3] T. Bjork, A. Murgoci, A general theory of Markovian time inconsistent stochastic control problems, Stockholm School of Economics (Working Paper), 2010 (2010), 55 pages. 3

[4] J.-F. Boulier, S. J. Huang, G. Taillard, Optimal management under stochastic interest rates: the case of a protected defined contribution pension fund, Insurance Math. Econom., 28 (2001), 173-189. 1

[5] A. J. G. Cairns, D. Blake, K. Dowd, Stochastic lifestyling: optimal dynamic asset allocation for defined contribution pension plans, J. Econom. Dynam. Control, 30 (2006), 843-877. 1

[6] M. Di Giacinto, S. Federico, F. Gozzi, Pension funds with a minimum guarantee: a stochastic control approach, Finance Stoch., 15 (2011), 297-342. 1

[7] J. W. Gao, Stochastic optimal control of DC pension funds, Insurance Math. Econom., 42 (2008), 1159-1164. 1

[8] J. W. Gao, Optimal portfolios for DC pension plans under a CEV model, Insurance Math. Econom., 44 (2009), 479-490. 1

[9] L. He, Z. X. Liang, Optimal financing and dividend control of the insurance company with fixed and proportional transaction costs, Insurance Math. Econom., 44 (2009), 88-94. 3

[10] L. He, Z. X. Liang, The optimal investment strategy for the DC plan with the return of premiums clauses in a mean-variance framework, Insurance Math. Econom., 53 (2013), 643-649. 1, 3

[11] D. P. Li, X. M. Rong, H. Zhao, B. Yi, Equilibrium investment strategy for DC pension plan with default risk and return of premiums clauses under CEV model, Insurance Math. Econom., 72 (2017), 6-20. 1

[12] Z.X. Liang, J. P. Huang, Optimal dividend and investing control of an insurance company with higher solvency constraints, Insurance Math. Econom., 49 (2011), 501-511. 3

[13] B. O. Osu, E. E. Akpanibah, C. Olunkwa, Mean-Variance Optimization of portfolios with return of premium clauses in a DC pension plan with multiple contributors under constant elasticity of variance model, Int. J. Math. Comput. Sci., 12 (2018), 85-90. 1

[14] B. O. Osu, E. E. Akpanibah, B. I. Oruh, Optimal investment strategies for defined contribution (DC) pension fund with multiple contributors via Legendre transform and dual theory, Int. J. Pure Appl. Res., 2 (2017), 97-105. 1

[15] D.-L. Sheng, X. M. Rong, Optimal time consistent investment strategy for a DC pension with the return of premiums clauses and annuity contracts, Discrete Dyn. Nat. Soc., 2014 (2014), 13 pages. 1, 3, 4, 7

[16] J. W. Xiao, Z. Hong, C. L. Qin, The constant elasticity of variance (CEV) model and the Legendre transform-dual solution for annuity contracts, Insurance Math. Econom., 40 (2007), 302-310. 1

[17] Y. Zeng, Z. F. Li, Optimal time consistent investment and reinsurance policies for mean-variance insurers, Insurance Math. Econom., 49 (2011), 145-154. 3

[18] C. B. Zhang, X. M. Rong, Optimal investment strategies for DC pension with astochastic salary under affine interest rate model, Discrete Dyn. Nat. Soc., 2013 (2013), 11 pages. 1 